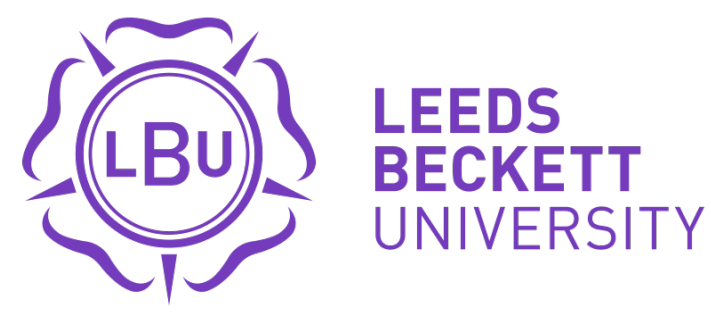

Citation:

Surr, CA and Jones, S and Hamilton, J (2016) Monitoring and improving the quality of personcentred care in health and social settings using Dementia Care Mapping (DCM): Staff experiences of implementation barriers and supports. European Journal for Person Centered Healthcare, 4 (1). pp. 17-23. ISSN 2052-5656 DOI: https://doi.org/10.5750/ejpch.v4i1.1042

Link to Leeds Beckett Repository record:

https://eprints.leedsbeckett.ac.uk/id/eprint/1415/

Document Version:

Article (Accepted Version)

The aim of the Leeds Beckett Repository is to provide open access to our research, as required by funder policies and permitted by publishers and copyright law.

The Leeds Beckett repository holds a wide range of publications, each of which has been checked for copyright and the relevant embargo period has been applied by the Research Services team.

We operate on a standard take-down policy. If you are the author or publisher of an output and you would like it removed from the repository, please contact us and we will investigate on a case-by-case basis.

Each thesis in the repository has been cleared where necessary by the author for third party copyright. If you would like a thesis to be removed from the repository or believe there is an issue with copyright, please contact us on openaccess@leedsbeckett.ac.uk and we will investigate on a case-by-case basis. 


\section{Monitoring and improving the quality of person-centred care in health and social settings using Dementia Care Mapping (DCM): Staff experiences of implementation barriers and supports}

Stephanie Jones, BA (hons) Research Assistant ${ }^{1}$, Janine Hamilton BA (hons) Project Manager $^{1}$, and Claire Surr PhD Professor ${ }^{2}$

${ }^{1}$ School of Dementia Studies

Faculty of Health Studies

University of Bradford

Bradford

BD7 1DP

UK

${ }^{2}$ School of Health and Community Studies

Faculty of Health and Social Sciences

Leeds Beckett University

Leeds,

LS13HE

UK

Address for correspondence:

Professor Claire Surr

Professor of Dementia Studies

Faculty of Health and Social Sciences

Portland 512, City Campus

Leeds Beckett University

Leeds,

LS1 3HE

UK

Email: c.a.surr@leedsbeckett.ac.uk

Tel: +44(0)1138124316

Suggested running title: Quality monitoring and improvement with DCM

Key words: Dementia, Dementia Care Mapping, care quality improvement, implementation, health, social care 


\section{Abstract}

Dementia Care Mapping (DCM) is a person-centred care quality monitoring and improvement tool used for nearly 20 years in formal dementia care settings and there are thousands of people trained in its use internationally. However, little is known about if and how DCM is used in practice or regarding application of interrater reliability checks to ensure data quality. This study aimed to explore the application of DCM in practice in the UK. A mixed methods design including an online survey and in-depth, semi-structured interviews was employed. There were 98 survey respondents, representing 71 health and social care, University/research and training/consultancy organisations. Twenty-one people participated in the semistructured interviews. Fifty-nine percent of survey respondents had used DCM since completing training. Those working in clinical roles and in health and social care provider organisations were least likely to have used DCM. A range of barriers and supports to use of DCM in practice after training were reported, including lack of management support and time. The majority of those who had used DCM also conducted inter-rater reliability checks at least once per year or more frequently. Of the $20 \%$ who did not the main barriers to this were absence of someone to conduct the checks with and lack of time and resources. The study has shown that it is possible to apply an evidence-based quality monitoring and improvement tool such as DCM to support implementation of person-centred care in practice, despite widespread limitations on time and resources as well as identifying a range of setting conditions that can help or hinder implementation. 


\section{Introduction}

The quality of care provided to people with dementia is an area of international concern (1). Tools and processes, which can effectively help practitioners to gather evidence to monitor and improve the quality of care with a particular focus on person-centred care are, therefore, required.

\section{Dementia Care Mapping}

Dementia Care Mapping (DCM)(2) is an observational tool, which has been used for the last 20 years, by practitioners working in a range of formal settings such as care homes, hospital wards and day centres, to measure and improve the quality of dementia care. DCM is grounded in the philosophy of person-centred care (3), which recognises the importance of understanding the unique experiences and needs of each individual with dementia. Therefore, its application is said to help services to move from provision of task focussed care primarily driven by the needs of the service, to a person-centred approach where the person with dementia is at the heart of provision. DCM is recommended within UK national guidelines as a method for supporting evidence-based, individualised care planning (4) and as an audit tool to measure quality of life for people with dementia (5).

DCM is both a tool and a practice development process, which includes a cycle consisting of five-phases (6): briefing, mapping, data analysis, feedback and action planning. The briefing phase involves trained DCM users (mappers), who usually observe (map) in pairs, meeting with staff in the facility where DCM will be used, to explain what DCM is and what the mapping process will involve. During the observation phase (mapping) mappers observe between one and eight people with dementia (participants) continuously over a period of time. Each mapper observes different participants so evidence-based observations on up to sixteen people with dementia can be included in any one DCM cycle. The length of observation is dependent on the purpose of the map and can range from short focused maps of 30 60 minutes to longer maps of 4-6 hours or more. Observations only take place in communal areas such as lounges, dining rooms, corridors and outside spaces and personal or private care is never observed. 
DCM observations are divided into five-minute periods (time frames). At the end of each five-minute time frame, the mapper records two pieces of information which describe the experience of each participant. The Behaviour Category Code (BCC) is an alphabetical code chosen from a list of 23 behavioural descriptions, including interacting verbally or non-verbally $(A)$, eating or drinking $(F)$, and engaging in reminiscence $(\mathrm{G})$. The participant's relative state of mood and engagement (Mood/Engagement Value (ME Value)) is also coded using a 6-point scale (-5 extreme negative state to +5 extreme positive state). Two additional data items are recorded by the mapper as and when they occur. Personal detractions (PDs) are staff behaviours that have the potential to undermine the participant or their wellbeing and are chosen from a list of 17 different behaviour types, including treating the person like an object (objectification) or speaking to them as if they were a child (infantilisation). Personal Enhancers (PEs) are the opposite of PDs and are thus staff behaviours that have the potential to support the participant, enhance their wellbeing or demonstrate person-centred practices and include providing physical or emotional comfort (warmth) and entering into a participants frame of reference (validation).

The analysis phase of the cycle involves processing of the raw DCM data into a feedback report in a format suitable for use by people not trained to use DCM. The feedback phase involves sharing the findings of the map with the staff team via one or more formal feedback sessions. Following on from this a number of action plans are developed by the staff team, with the purpose of implementation over an agreed period. The DCM cycle is then repeated at regular intervals (usually every 4-12 months) to monitor progress and identify new action plans.

DCM training is available in a number of countries internationally. To learn to use the DCM tool, attendance at a 3-4 day 'basic user' course is required (6). Following on from basic user training mappers can increase their expertise through attending 'advanced user' training; the highest level of expertise possible being 'DCM trainer'. Since its inception DCM has been used for a range of purposes including: at an individual level for care planning(7); at a setting level for care quality monitoring and 
improvement and identification of staff training and development needs (8); at an organisational level to provide an overall care quality audit (9); and as a research outcome measure for assessment of quality of care and quality of life (10).

There is a growing body of evidence regarding the effectiveness of DCM as a practice development tool and process. Practitioners report finding DCM a valuable tool for improving well-being in people with dementia (10) and supporting the monitoring and improvement of person-centred care $(11,12)$. A number of studies conducted in care home settings, indicate that compared to usual care controls, use of DCM leads to a reduction in resident agitation, anxiety (13-16) and other neuropsychiatric symptoms (16), depression (14) and falls (15) and improved resident quality of life (16). DCM also leads to increased feelings of staff connectedness with (13) and positivity towards residents (17), improved quality of staff interactions (14) and reduced emotional exhaustion and staff burnout (18). There remains limited available evidence on the cost-effectiveness of DCM.

DCM has been criticised for being a complex tool and studies have, therefore, suggested a lack of coding consistency between mappers is of concern, raising concerns about the potential usefulness DCM data as a robust evidence-based measure of care quality or quality of life (19). DCM guidance (2) recommends that mappers establish inter-rater reliability (IRR) (minimum $70 \%$ concordance across $\mathrm{BCC}$ and ME values) with any co-mapper ahead of use, and that every mapper conduct an IRR map at least once per year. Published literature highlights that while a number of research studies have examined IRR and demonstrated it can be established effectively between experienced mappers in research contexts $(10,20)$, relatively little is really known about IRR between mappers in everyday practice or whether IRR checks are completed regularly (19). In one study, Thornton et al examined IRR in the context of everyday mapping. They found that for 12 BCC's IRR of lower than $50 \%$ was achieved and appropriate IRR (70\%) was not obtained for any of the ME values. The paper does not include information on the regularity of IRR checks between mappers prior to participating in this study. Little is also known about how closely trained mappers adhere to DCM guidance on IRR frequency. 
The implementation of DCM as an evidence-based quality monitoring and practice development tool

There are, currently, thousands of people trained in DCM internationally (21). However, little is currently known about how many actually use DCM in practice, how often, whether they conduct regular IRR checks or what the main barriers and supports for DCM implementation are. The findings of two existing surveys of DCM users conducted in the late 2000s, in the UK $(n=79)$ and the US $(n=82)(21)$ found that around $30 \%$ of basic level users in the US and $50 \%$ in the UK had mapped since training. The key challenges to use of mapping in practice by respondents in both countries were a lack of time, lack of organisational backing and issues with delays in being able to conduct feedback sessions with the staff team. Despite these issues, around $60 \%$ of respondents from both countries felt attending DCM training had helped them to develop a more positive attitude towards people with dementia and to subsequently develop a more person-centred outlook. There are limitations to these surveys; both were conducted a considerable period of time ago and therefore may not be representative of contemporary implementation patterns or issues affecting mappers. The sampling method within both surveys included disproportionate numbers of mappers trained to an advanced user level, meaning a bias towards inclusion of mappers who had mapped, since completion of a map is part of advanced user training. Both surveys also included largely quantitative items, meaning explanations for particular findings were not able to be explored further.

\section{Aims}

This study therefore aimed to understand whether, in the current UK health and social care system, trained mappers are implementing DCM in practice, whether they are conducting regular IRR checks and what the current barriers and supports to implementation of DCM in practice are.

\section{Methods}


A mixed-methods design was employed involving a survey of DCM users with a series of follow-up in-depth interviews.

\section{Survey}

An on-line survey containing 28 questions covering the areas of participant demographics, DCM training and use was administered using 'Survey Monkey', although respondents could request an e-mail or paper version to complete if they preferred. A mix of open and closed questions was used to provide the opportunity to gather more detailed reasons for particular answers. The survey was piloted with three volunteers who were experienced DCM users to ensure questions were suitable and easy to understand and modifications to question wording was made based on their feedback. The survey was open for 4 weeks. Survey respondents were asked to give their name and contact details as part of the survey, although they could choose to leave this blank of they wished and participants were informed that their personal details would remain anonymous for analysis purposes.

Recruitment to the survey was undertaken via a range of approaches including sending e-mail invites to a sample of 679 UK based, DCM users for whom there were e-mail contact details available on the database held at the University of Bradford. The survey was also promoted via the University of Bradford web-site and via social media such as Facebook and Twitter. To maximise response rates we followed best-practice guidelines for web-based surveys (22) including personalised invite and follow-up e-mails and offered incentives in the form of dementia related book prizes.

\section{Interviews}

As part of the survey respondents were asked if they would be willing to take part in a follow-up interview. Interviews were designed to follow-up on the responses gained on the survey with the aim of understanding the issues leading to some of the survey findings. Therefore a representative sample of survey respondents who said they would be willing to participate in a follow-up interview were approached. The interview schedule consisted of 51 questions for respondents who had conducted mapping at least once since undergoing training and 29 questions for participants 
who had not conducted mapping since undergoing training. Questions were based on key issues arising from the survey data. Interviews were conducted by telephone or Skype at a time convenient to the interviewee. Interviews were audio recorded. Audio recordings were listened to again and responses to each question were summarised in written format, alongside transcription of key verbatim quotes for the purposes of analysis.

\section{Ethical issues}

Ethical approval for the study was granted by the University of Bradford Research Ethics Panel. Survey participants had given consent for their details to be held on the University of Bradford database and to be contacted regarding DCM related issues. Completion of the survey was voluntary. Interview participants provided written informed consent for the interviews to take place and to be audio recorded.

\section{Data analysis}

Data were extracted from the survey tool into a Microsoft Excel spreadsheet and then into IBM SPSS version 22 and analysed using descriptive statistics. Interview data was analysed using framework analysis, a method appropriate for use when the research aims and questions are particularly focussed (Smith and Firth, 2011). Framework analysis involves a process of: becoming familiar with the data; summarising and synthesising the data through refining initial themes and codes leading to emergence of the whole picture and key concepts; finding meaning in the data through interpretation of themes and core concepts and seeking their wider application.

\section{Results}

\section{Survey Respondents}

Ninety-eight participants completed the survey. Participants' demographical details can be found in table 1 . Eighty-three (87\%) were female and the majority worked in a care homes setting $(43 \%)$ or in the NHS $(38 \%)$, with smaller numbers working in the community $(6 \%)$, a university or research institute $(7 \%)$ or in a training/consultancy organisation (5\%). Participants represented 71 different organisations in total. Most participants worked in direct care roles $(51 \%)$, management $(21 \%)$ or training roles 
$(15 \%)$ with the remainder working in quality monitoring $(9 \%)$ or research $(4 \%)$ focussed positions. The majority of respondents $(78 \%)$ were trained to DCM basic user level. A significant minority of respondents did not provide details of how long ago they attending training, although of those who did, the majority (56\%) completed their training within the last four years.

\section{Interview participants}

Twenty-one participants took part in the semi-structured interviews. Thirteen participants $(62 \%)$ worked in care home provider organisations and nine $(43 \%)$ in the NHS. The majority worked in direct care/clinical roles $(62 \%)$, with smaller numbers in managerial (19\%), training (14\%) and quality monitoring/assurance $(5 \%)$ roles. Ten of those interviewed had used DCM in practice (48\%) and 11 (52\%) had not.

[insert table 1 about here]

\section{Regularity of DCM use}

Of the 81 survey respondents who answered the question, $59 \%$ had mapped since training and $41 \%$ had never used DCM in practice. Of those who had mapped, $28 \%$ completed 1-2 maps per year, 11\% 3-4 maps per year, 10\% 5-6 maps per year and $10 \%$ conducted more than 6 maps per year. Ninety percent $(n=19)$ of those who took part in the semi-structured interviews stated they expected to use DCM back in their workplace on completion of DCM training, indicating that non-use of DCM does not appear to be related to people attending training who have no intention to use the tool. Of the 11 interview participants who had not mapped, five (45\%) hoped to use mapping in the next six-months while six $(55 \%)$ said they had no plans to map. None of the interview respondents who had previously mapped said they would definitely not be conducting at least one map in the next 6 months, with $90 \%$ stating they had plans to map and $10 \%$ unsure.

We have started practising to get our results, sort of matching, and then we will be mapping properly ... officially. (Participant 2)

Of the 79 survey respondents who answered the question, direct care/clinical staff were the least likely to have ever mapped with $55 \%$ of those working in this type of 
role saying they had never mapped. Those working in a quality monitoring and improvement role were most likely to have mapped with all respondents $(100 \%)$ in this role having mapped since completing DCM training, followed by those working in training roles $(82 \%)$, management roles $(63 \%)$ and research $(50 \%)$. In terms of mapping frequency, those in a quality monitoring and improvement role mapped most frequently with $29 \%$ reporting conducting 6 or more maps per year and a further $29 \%$ saying they conducted 3-4 per year. Those working in direct care/clinical roles most often conducted 1-2 maps per year (23\%). However, $10 \%$ working in this type of role said they mapped more than six times a year. Of the researchers who had mapped (50\%) all of them conducted only $1-2$ maps a year. The majority $(55 \%)$ of those working in training roles said they mapped once or twice a year.

In terms of organisation type and regularity of mapping, those working within community care or support organisations were the least likely survey respondents to have mapped, with all (100\%) respondents saying they had not used DCM since training. Those working in a training/consultancy organisation were most likely to have mapped $(80 \%)$ followed by those working in a university/research institute $(75 \%)$ and care home provider $(61 \%)$. Just over half $(57 \%)$ of respondents who worked in the NHS had ever mapped. With regard to frequency of mapping, $13 \%$ of NHS staff mapped more than six times per year, $20 \%$ of those in training/consultancy organisations mapped this frequently, while only $8 \%$ of those working in care home provider organisations mapped more than six times per year. No respondents who worked in a university/research institute said they mapped more than four times per year.

The survey data indicated a range of reasons why people had not mapped including needing someone else to map with (22\%), lack of opportunity (22\%), lack of management support (16\%), change of job (13\%), lack of time (9\%) and DCM being unsuitable for role or setting in which the person worked (12\%). Of semi-structured interview participants, $80 \%$ of those mapped stated there was full organisational support for use of DCM and $20 \%$ said there had previously been support but this was no longer present due to the DCM lead leaving the organisation or to demands on time and resources. Fifty-five percent of those who had never mapped said there 
was organisational support for DCM and all five interviewees who had not mapped but said they planned to in the next six-months, worked in organisations that supported DCM. Sixteen (76\%) of those who were interviewed, whether they had ever mapped, stated that having more staff and resources would help to increase the amount of mapping activity in their organisation.

Interviewer: Is there anything that would help to increase the amount of mapping that you do?

Participant: Time

Interviewer: Anything else or just time?

Participant: Just time.

(Participant 9)

\section{Inter-rater reliability}

Over half $(53 \%)$ of the 79 survey respondents to this question said they had never conducted an inter-rater reliability check. However, once those who had never mapped were removed from this data the majority of respondents conducted IRR checks at least once per year (44\%) or more frequently (36\%). Only $20 \%$ of those who had mapped had never conducted an IRR check and the main reasons given by survey respondents for this were a lack of availability of someone else to conduct an IRR check with (55\%), lack of time (22\%), no opportunity (11\%) and lack of awareness that IRR should be checked (11\%). While people were more likely to have conducted IRR than not, direct care/clinical staff had the highest proportion of staff who had used DCM but who had never conducted IRR $(28 \%)$ followed by those in training (22\%) and quality monitoring and improvement (17\%) roles.

Semi-structured interview respondents indicated lack of time and resources were a barrier to conducting regular IRR. Of those who had mapped, 30\% said their organisation did not support them to conduct IRR checks and a further $30 \%$ said that while it was supported, time was a factor in whether it was carried out. Only one respondent (10\%) said IRR was both supported and monitored at an organisational level. Of those who had never mapped, one (9\%) said IRR would not be supported by their organisation and two (18\%) were unsure. Two respondents (18\%) who had 
not mapped said they did not have a colleague they could conduct IRR with in the future.

Interviewer: Would you say IRR is supported in your organisation? Participant: No! They don't know what l'm talking about. They don't understand it.

(Participant 6)

Only $9 \%$ of those in management roles who responded to the survey had never conducted IRR despite mapping and all researchers had conducted IRR. Those in management roles were most likely to conduct IRR more frequently with $27 \%$ stating they complete IRR more than twice a year and $9 \%$ stating more than once per month. Most of those who conducted IRR did so once per year. Around one quarter of those working in the NHS and in care home organisations who had mapped, had never conducted IRR (25\% and $23 \%$ respectively). All those employed in training/consultancy and university/research institute organisations had conducted IRR, however no-one working in these types of organisations conducted IRR more than twice per year. Thirty-one percent of those in the NHS and $14 \%$ of those employed by care home provider organisations conducted IRR more than twice per year.

\section{Discussion}

As with a previous surveys of DCM users (21), this study found that a number of those trained do not go on to use DCM afterwards. This study showed a slightly higher percentage of respondents who had used DCM $(60 \%)$ than in previous studies (50\% in UK and $30 \%$ in US). Previous surveys have not asked about mapping intentions in the future and this study indicated that around $30 \%$ of those who had not mapped had intentions to do so in the next six-months. Clearly intentions do not equate to actual practice, however, it remains a positive finding that a proportion of those who have not mapped remain committed to mapping in the future. 
As with previous surveys, respondents to this survey as well as participants in the semi-structured interviews indicated that one of the main barriers to use of DCM in practice was time and resources. Increased time and resources were identified by the vast majority of mappers as being something that would increase mapping activity in their organisation, whether or not they had previously mapped, indicating that this remains an issue even in settings where mapping does occur. A lack of time and resources is commonly cited within health and social care as a barrier to delivery of person-centred care and to the utilisation of quality monitoring and improvement tools and processes (21). This study shows that despite limited resources some organisations are able to support regular DCM activity. Further, within these organisations, exploring the processes and supportive mechanisms they employ to achieve this will be beneficial to understanding the setting conditions required to support implementation of quality monitoring and improvement tools in practice.

A key finding within this study was that organisational support for DCM was required for its implementation in practice. No mappers who participated in semi-structured interviews indicated that mapping was possible where there was not organisational support. Conversely of those who had not mapped, all those who indicated they had plans to use the tool within the next six-months stated they had organisational support for mapping. Those trained in DCM who felt there was no organisational support for its implementation, indicated they had no plans to conduct DCM in the following six-months. These results are a clear indicator that it is only possible to implement DCM in an organisation where it is supported from the top down.

A further barrier to DCM implementation was lack of someone else to map with. The DCM guidance recommends but does not require users to map in pairs on the basis that working with someone else provides support throughout the process and is more likely to produce a better quality outcome. In particular having someone to work with during the delivery of feedback sessions and in the action planning process with staff, can be helpful, particularly if the results of the observations may prove to be challenging for staff to engage with. This is not an issue that has been identified in previous literature as being a barrier to DCM implementation. 
This was the first study to examine use of DCM and its relationship to mapper jobrole and type of organisation employed in. The results of the survey indicated that those most likely to use DCM worked in roles that were non-practice/clinically focussed. In particular, those in training and quality monitoring and improvement roles, while making up a minority of respondents, were most likely to map. Over half of staff in practice/clinical roles had never mapped indicating it seems particularly difficult for staff in such roles to be able to find or be supported to have the time to use DCM. Health and social care provider organisations, therefore, need to consider issues such as availability to release staff to use DCM ahead of making decisions about which staff should receive training.

The percentage of staff who have mapped based on employer types are perhaps unsurprising, given the information about mapping frequency within particular job roles. Training/consultancy and university/research institute organisations were most likely to be able to support their staff to conduct DCM, however such respondents made up a minority of the survey sample. Around $60 \%$ of respondents from care homes and the NHS had mapped. Interestingly, despite having the lowest percentage of those trained in DCM who had actually mapped, NHS staff made up a significant proportion of those who used DCM very frequently (6 or more times per year). Further research into the ways in which DCM is being used in organisations where mapping occurs frequently is needed, as these organisations are supporting its regular use despite the time and resources needed to do so. They may provide strong indicators of the benefits of evidence-based quality monitoring and improvement tools, which can be shared with the sector.

Any quality monitoring and improvement tool is only as good as the data it is based upon and IRR is one approach to ensuring accuracy and consistency of data across an organisation with many DCM users, and over time. The need to establish IRR between DCM users has been discussed by a number of previous studies $(10,11$, $20)$, however, this is the first study, to examine if those trained in DCM are conducting regular IRR maps, if so how frequently and if not what the barriers to this are. Encouragingly, of those who had mapped only $20 \%$ never carried out IRR checks with the biggest barrier to doing this being a lack of someone else to 
complete IRR with. Some mappers who took part in semi-structured interviews indicated that their organisation was not always supportive of IRR being conducted largely due to time and resource implications. A number of mappers reported that IRR was fully integrated into the way their organisation used DCM and, therefore, further research into how this has been achieved would be beneficial to understand and share best practice.

\section{Limitations}

This was a fairly small sample size and due to lack of availability of demographic data on the trained DCM user population, it is not possible to be sure of the representativeness of the sample compared to the population as a whole. The small size of sub-groups did not permit analysis of data using techniques other than descriptive statistics. However, the sample size is the largest to date for a published survey of DCM users. The survey used a convenience sample and participants were recruited via e-mail invite and advertising on web-sites and social media. Therefore, this may have restricted the recruitment of mappers who do not actively engage with social media or IT.

\section{Conclusions}

Overall the study has shown that it is possible to implement an evidence-based quality monitoring and improvement tool such as DCM in practice, despite widespread limitations on time and resources. It has also provided information about the setting conditions that can help with implementation or issues that can serve as barriers to this. 


\section{Acknowledgements}

The study was funded by the University of Bradford Research and Knowledge Transfer Strategic Development Fund. We would like to thank the survey respondents and interview participants who freely gave of their time to provide data for this study. 


\section{References}

1. WHO/Alzheimer's Disease International. Dementia: A Public Health Priority. London: WHO, 2012.

2. Bradford Dementia Group. DCM 8 User's Manual. Bradford: University of Bradford; 2005.

3. Brooker D, Surr C. Dementia Care Mapping (DCM): initial validation of DCM 8 in UK field trials. International Journal of Geriatric Psychiatry. 2006;21(11):1018-25.

4. NICE/SCIE. Dementia. Supporting people with dementia and their carers in health and social care. NICE clinical guideline 42. London: British Psychological Society and Royal College of Psychiatrists, 2006.

5. National Audit Office. Improving dementia services in England - an Interim Report. London: National Audit Office, 2010.

6. BSI. PAS 800:2010. Use of Dementia Care Mapping for improved person-centred care in a care provider organization. Guide. London: BSI; 2010.

7. Packer T. Haloperidol, hips and toenails... Journal of Dementia Care. 1997(Nov/Dec):22-4.

8. Younger D, Martin GW. Dementia care mapping: an approach to quality audit of services for people with dementia in two health districts. Journal of Advanced Nursing. 2000;32(5):1206-12.

9. Brooker D, Foster N, Banner A, Payne M, Jackson L. The efficacy of Dementia Care Mapping as an audit tool: report of a 3-year British NHS evaluation. Aging and Mental Health. 1998;2(1):60-

70.

10. Brooker D. Dementia Care Mapping: A Review of the Research Literature. Gerontologist. 2005;45(Special Issue 1):11-8.

11. Beavis D, Simpson S, Graham I. A literature review of Dementia Care Mapping: methodological considerations and efficacy. Journal of Psychiatric and Mental Health Nursing. 2002;9:725-36.

12. Mansah M, Coulon L, Brown P. A mapper's reflection on Dementia Care Mapping with older residents living in a nursing home. International Journal of Older People Nursing. 2008;3:113-20.

13. Kuiper D, Dijkstra GJ, Tuinstra J, Groothoff JW. The influence of Dementia Care Mapping (DCM) on behavioural problems of persons with dementia and the job satisfaction of caregivers: a pilot study. Tijdschrift voor Gerontologie en Geriatrie. 2009;40(3):102-12.

14. Chenoweth $\mathrm{L}$, Jeon $\mathrm{YH}$. Determining the efficacy of dementia Care Mapping as an outcome measure and process for change: A pilot study. Aging and Mental Health. 2007;11(3):237-45.

15. Chenoweth L, King MT, Jeon YH, Brodaty H, Stein-Parbury J, Haas M, et al. Caring for Aged Dementia Care Resident Study (CADRES) of person-centred dementia care, dementia-care mapping, and usual care in dementia: a cluster-randomised trial. The Lancet - Neurology. 2009;8(April):317-25. 16. Rokstad AM, Røsvik J, Kirkevold $\varnothing$, Selbæk G, Saltyte Benth J, Engedal K. The Effect of Person-Centred Dementia Care to Prevent Agitation and Other Neuropsychiatric Symptoms and Enhance Quality of Life in Nursing Home Patients: A 10-Month Randomized Controlled Trial. Dementia and Geriatric Cognitive Disorders. 2013;36:340-53.

17. van de Ven G, Drasovic I, Adang EMM, Donders R, Zuidema S, Koopmans RTCM, et al. Effects of Dementia-Care Mapping on Residents and Staff of Care Homes: A Pragmatic Cluster-Randomised Controlled Trial. PLoS ONE. 2013;8(7):e67325.

18. Jeon YH, Luscombe G, Chenoweth L, Stein-Parbury J, Brodaty H, King M, et al. Staff outcomes from the Caring for Aged Dementia Care REsident Study (CADRES): A cluster randomised trial. International Journal Nursing Studies. 2012;49(5):508-18.

19. Thornton A, Hatton C, Tatham A. Dementia Care Mapping reconsidered: exploring the reliability and validity of the observational tool. International Journal of Geriatric Psychiatry. 2004;19:718-26.

20. Cooke HA, Chaudhury H. An examination of the psychometric properties and efficacy of Dementia Care Mapping. Dementia. 2012;12(6):790-805. 
21. Douglass C, Keddie A, Brooker D, Surr C. Cross-Cultural comparison of the perceptions and experiences of Dementia Care Mapping "Mappers" in the United States and the United Kingdom. Journal of Aging and Health. 2010;22(5):567-88.

22. Sánchez-Fernández J, Muñoz-Leiva F, Montoro-Ríos FJ. Improving retention rate and response quality in Web-based surveys. Computers in Human Behaviour. 2012;28:507-14. 
Figure legends

Table 1: Participant demographics 
Table 1: Participant demographics

\begin{tabular}{lll}
\hline Demographic (n) & Characteristic & $\%$ \\
\hline Gender (95) & Male & 13 \\
Organisation type (94) & Female & 87 \\
& Care home & 43 \\
& NHS & 38 \\
& Community & 6 \\
& Training/consultancy & 5 \\
& University/Research Institute & 7 \\
Role (94) & Direct care delivery & 51 \\
& Management & 21 \\
& Training & 15 \\
& Research & 4 \\
Level of DCM training & Quality monitoring and implementation & 9 \\
(81) & Basic user & 78 \\
& Advanced User & 9 \\
Length of time since last & Higher level user & 4 \\
DCM training (98) & Less than 1 year & 7 \\
& $1-2$ years & 13 \\
& 3-4 years & 23 \\
& 5-9 years & 19 \\
& 10 years and over & 8 \\
& Not known & 1 \\
\hline
\end{tabular}

